Pesq. Vet. Bras. 37(4):355-358, abril 2017

DOI: $10.1590 /$ S0100-736X2017000400009

\title{
Potencial evocado visual em cães e ovinos ${ }^{1}$
}

\author{
Mariana Isa Poci Palumbo², Luiz Antonio de Lima Resende ${ }^{3}$, Rodrigo Barros ${ }^{4}$, \\ César Erineudo Tavares de Araújo ${ }^{2}$, Cláudia Valéria Seullner Brandão ${ }^{4}$ \\ e Alexandre Secorun Borges ${ }^{2 *}$
}

\begin{abstract}
Palumbo M.I.P., Resende L.A.L., Barros R., Araújo C.E.T., Brandão C.V.S. \& Borges A.S. 2017. [Visual evoked potential in dogs and sheep.] Potencial evocado visual em cães e ovinos. Pesquisa Veterinária Brasileira 37(4):355-358. Departamento de Clínica Veterinária, Faculdade de Medicina Veterinária e Zootecnia, Universidade Estadual Paulista Júlio de Mesquita Filho, Distrito de Rubião Junior s/n, Botucatu, SP 18618-970, Brazil. E-mail: palumboma11@yahoo.com.br

The visual evoked potential (VEP) is an electro-diagnostic technique that allows assessment of visual pathways. The VEP from 20 adult dogs and 20 adult sheep of both gender was obtained for normative study. Monopolar electrode needles were placed in $\mathrm{O}_{1}$ and $\mathrm{O}_{2}$ (active), $\mathrm{F}_{\mathrm{pz}}$ (common reference) and $\mathrm{C}_{\mathrm{z}}$ (ground). The stimuli were flash at $1 \mathrm{~Hz}$. Seventeen dogs and 17 sheep were included in the study because they had auditory potentials with good technical quality. The results from both eyes were grouped, totaling 34 results in each species. The most consistent result in all tests was an electropositive deflection, with a mean latency $55.4 \mathrm{~ms}$ in dogs (P55) and $63.75 \mathrm{~ms}$ in sheep (P63). Two negative deflections were also identified: N31 and N75 in dogs, and N42 and N86 in sheep. The methodology used in this study allowed to obtain potential similar to those described in other studies, with little variability, and is useful for evaluation of animals with suspected changes in the central visual pathways.
\end{abstract}

INDEX TERMS: Visual evoked potential, neurology, oftalmology, dog, sheep.

RESUMO.- O potencial evocado visual (PEV) é técnica eletrodiagnóstica que permite avaliação das vias visuais. 0 PEV de 20 cães e 20 ovinos adultos, de ambos os sexos, foram obtidos para estudo normativo. Foram utilizados eletrodos de agulha monopolar posicionados em $\mathrm{O}_{1} \mathrm{e}$ $\mathrm{O}_{2}$ (ativos), em $\mathrm{F}_{\mathrm{pz}}$ (referência comum) e em $\mathrm{C}_{\mathrm{z}}$ (terra). 0 estímulo foi na forma de flash a $1 \mathrm{~Hz}$. Dezessete cães e 17 ovinos foram incluídos no estudo, pois apresentaram potenciais auditivos com boa qualidade técnica. Os resultados da avaliação dos dois olhos foram agrupados, totalizando

\footnotetext{
${ }^{1}$ Recebido em 2 de abril de 2015.

Aceito para publicação em 10 de março de 2016.

${ }^{2}$ Departamento de Clínica Veterinária, Faculdade de Medicina Veterinária e Zootecnia (FMVZ), Universidade Estadual Paulista "Júlio de Mesquita Filho" (Unesp), Distrito de Rubião Junior s/n, Botucatu, SP18618-970, Brasil. E-mail: palumboma11@yahoo.com.br; *Autor para correspondência: asborges@fmvz.unesp.br

${ }^{3}$ Departamento de Neurologia, Psicologia e Psiquiatria, Faculdade de Medicina de Botucatu (FMB), Unesp, Distrito de Rubião Junior s/n, Botucatu, SP, Brasil 18618-970. E-mail: luan_resende@uol.com.br

${ }^{4}$ Departamento de Cirurgia e Anestesiologia Veterinária, FMVZ-Unesp, Distrito de Rubião Junior s/n, Botucatu, SP 18618-970, Brasil. E-mail: rodrigobarros@veterinario.med.br
}

34 exames em cada espécie. 0 achado mais consistente em todos os exames foi uma deflexão eletropositiva, com latência média de 55,4ms em cães (P55) e 63,75ms em ovinos (P63). Outras duas ondas eletronegativas também foram identificadas: N31 e N75 em cães; e N42 e N86 em ovinos. A metodologia utilizada neste estudo permitiu obtenção de potenciais visuais com pequena variabilidade, sendo útil para avaliação de animais com suspeita de alteração nas vias visuais centrais.

TERMOS DE INDEXAÇÃO: Potencial evocado visual, neurologia, oftalmologia, cão, ovino.

\section{INTRODUÇÃO}

Potencial evocado visual (PEV) é uma técnica eletrodiagnóstica utilizada para registrar a atividade elétrica das vias visuais após a retina (Kimotsuki et al. 2005). Os registros de PEV são feitos com eletrodos que captam a atividade elétrica gerada após estímulo visual (Sims et al. 1989). Os estímulos podem ser na forma de flash de luz ou padrão reverso alternado, que requer fixação do olhar no centro da tela. 0 resultado do exame consiste em deflexões positivas (P) e negativas $\mathrm{N}$ ), com diferentes latências e am- 
plitudes. Aumentos nas latências das ondas registradas no PEV podem indicar lesões nas vias visuais, sendo importantes para complementar o exame neurológico (Strain et al. 1986b).

As ondas registradas podem ser identificadas sequencialmente (P1, N1, P2, N2, P3) ou pelas latências em milissegundos com que aparecem (P40, N70 e P100, por exemplo) (Strain et al. 1986b). É provável que os geradores das atividades elétricas registradas nos potenciais evocados visuais sejam os mesmos em diferentes espécies de mamíferos, mas é necessária a obtenção de dados normativos para cada espécie e em cada laboratório, pois os resultados podem ser influenciados pelo tamanho da cabeça, dos olhos e do encéfalo, orientação dos geradores com relação aos eletrodos, presença ou ausência de chifres e pelo protocolo utilizado para registro (Strain et al. 1991b).

Em medicina humana, os PEV são rotineiramente utilizados e têm inúmeras aplicações clínicas, como para avaliar a evolução de esclerose múltipla, confirmar a condução do estímulo nas vias visuais ou mensurar a acuidade visual. Entretanto, o uso desta técnica em medicina veterinária ainda é pouco difundido (Loew 2005). Outros autores já descreveram a técnica de PEV para uso em cães (Boyer \& Kirk 1973, Bichsel et al. 1988, Sims et al. 1989, Kimotsuki et al. 2005, 2006), ovinos (Gregory \& Wotton 1983, Strain et al. 1986a, 1991a), suínos (Strain et al. 2006), gatos (Sims \& Laratta 1988, Imai et al. 1990), bovinos (Strain et al. 1986b, 1989, Takeuchi et al. 1993), ratos (Creel 1973) e equinos (Ström 2004, Michanek 2013), testando diferentes metodologias para realização do teste em diferentes espécies. 0 objetivo deste estudo foi registrar o PEV com flash em cães e ovinos clinicamente normais, para normatização da técnica e dos resultados para estas espécies.

\section{MATERIAL E MÉTODOS}

O Potencial evocado visual (PEV) foi obtido de 20 cães e 20 ovinos sem raça definida, de ambos os sexos, com idade entre 1 e 5 anos, após aprovação pelo Comitê de Ética em Experimentação Animal da FMVZ/Unesp-Botucatu (Processo 263/2011). Todos os animais foram submetidos ao exame oftalmológico, incluindo exames de fundo de olho. Apenas animais sem alterações oftálmicas foram incluídos no estudo.

Os PEV foram realizados no Laboratório de Eletroneurodiagnóstico, Faculdade de Medicina Veterinária e Zootecnia, Unesp-Botucatu. Os registros foram realizados sem sedação, sem uso de medicações midriáticas e sem adaptação ao escuro. Eletrodos de agulha monopolar foram posicionados em $\mathrm{O}_{1}$ e $\mathrm{O}_{2}$ (ativos), em $\mathrm{F}_{\mathrm{pz}}$ (referência comum) e em $\mathrm{C}_{\mathrm{z}}$ (terra). 0 posicionamento dos eletrodos foi baseado em outros estudos já realizados nestas espécies (Strain et al. 1986a, 1991a, Kimotsuki et al. 2005, 2006). A impedância dos eletrodos foi mensurada e mantida menor que $5 \mathrm{k} \Omega$. Foram utilizados filtros com banda passante de 10 a $30 \mathrm{~Hz}$, sensibilidades de $5 \mu \mathrm{V} /$ divisão e varredura de $20 \mathrm{~ms} /$ divisão. Todos os exames foram realizados em sala escura, com estímulo por flashes de luz com 0,5 J de intensidade, emitidos na frequência de $1 \mathrm{~Hz}$. A lâmpada foi mantida a $30 \mathrm{~cm}$ de distância dos olhos. 0 olho contralateral foi coberto por um material opaco, para que a estimulação fosse monocular. Em cada lado testado, foram obtidos dois traçados de no mínimo 100 promediações cada. 0 equipamento utilizado foi o Synergy Viasys Healthcare ${ }^{\circledR}$ de dois canais.

\section{RESULTADOS}

Não foi possível obtenção de registros de boa qualidade técnica em três cães e em três ovinos, pois eram animais mais agitados, cujos resultados mostraram muita interferência. Assim, os registros de 17 animais de cada espécie foram incluídos nos resultados finais. Não houve diferença significativa quando os resultados obtidos do lado esquerdo foram comparados com o direito (teste t não pareado, Graphpad software, $\mathrm{P}>0.05$ ). Assim, todos os resultados foram agrupados, totalizando 34 resultados para cada espécie. Em todos os exames o achado mais consistente foi uma onda eletropositiva ocorrendo com média de 55,4 ms em cães e 63,75 ms em ovinos (Fig.1). Também foi possível a identificação da deflexão negativa anterior e posterior à eletropositividade principal. As ondas foram nomeadas segundo a latência média: N31, P55 e N75 para cães e N42, P63 e N86 para ovinos. Também foram mensuradas duas amplitudes de ondas em $\mu$ V: N31-P55 e P55-N75. Os Quadros 1 e 2 mostram a média, o desvio padrão, os valores mínimo e máximo e intervalo de confiança a 95\% para as latências e amplitudes registradas para cães e ovinos, respectivamente.

\section{DISCUSSÃO}

As deflexões positivas, identificadas como P55 em cães e P63 em ovinos, são equivalentes ao pico P100 registrado em PEV de humanos. A P100 humana representa, provavelmente, a atividade elétrica cortical e é o principal achado utilizado para detecção de anormalidades nesta via sensorial (Chiappa 1990). Em outros estudos realizados em cães e ovinos, os autores identificaram três deflexões positivas e três negativas. Nos traçados do presente estudo também seria possível a identificação de 6 ondas, mas optou-se por

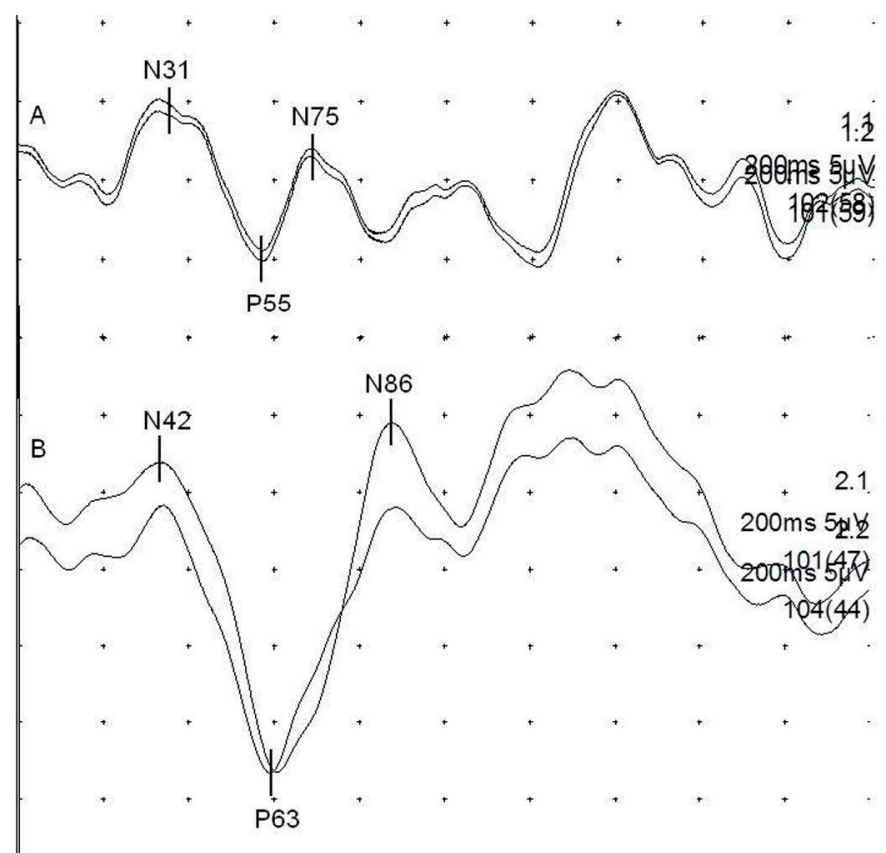

Fig.1. (A) Potencial evocado visual por flash de cão adulto. (B) Potencial evocado visual por flash de ovino adulto. Notar a identificação dos picos de onda registrados. Divisão vertical: amplitude $(5 \mu \mathrm{V})$. Divisão horizontal: latência (20 ms). 
Quadro 1. Valores de latência (ms) e amplitudes entre os picos $(\mu V)$ dos potenciais evocados visuais obtidos de cães $(n=34)$

\begin{tabular}{ccccc}
\hline Medida & Média & $\begin{array}{c}\text { Desvio } \\
\text { Padrão }\end{array}$ & $\begin{array}{c}\text { Mínimo e } \\
\text { Máximo }\end{array}$ & $\begin{array}{c}\text { Intervalo de } \\
\text { Confiança 95\% }\end{array}$ \\
\hline N31 & 31,34 & 4,69 & $18,6-41,2$ & $29,703-32,979$ \\
P55 & 55,4 & 5,88 & $45,8-65,0$ & $53,347-57,453$ \\
N75 & 75,2 & 13,10 & $59,8-103,4$ & $70,626-79,774$ \\
N31-P55 & 7,26 & 3,49 & $2,0-18,4$ & $6,046-8,483$ \\
P55-N75 & 5,62 & 3,07 & $1,3-13,6$ & $4,55-6,691$
\end{tabular}

Quadro 2. Valores de latência (ms) e amplitudes entre os picos $(\mu V)$ dos potenciais evocados visuais obtidos de ovinos $(n=34)$

\begin{tabular}{ccccc}
\hline Medida & Média & $\begin{array}{c}\text { Desvio } \\
\text { Padrão }\end{array}$ & $\begin{array}{c}\text { Mínimo e } \\
\text { Máximo }\end{array}$ & $\begin{array}{c}\text { Intervalo de } \\
\text { Confiança 95\% }\end{array}$ \\
\hline N42 & 42,3 & 9,7 & $26,2-55,2$ & $38,912-45,688$ \\
P63 & 63,75 & 4,21 & $56,6-70$ & $62,278-65,216$ \\
N86 & 86,82 & 9,6 & $74,8-111,8$ & $83,471-90,176$ \\
N42-P63 & 9,38 & 3,79 & $4,1-20,1$ & $8,053-10,70$ \\
P63-N86 & 10,96 & 4,41 & $2,7-20,0$ & $9,427-12,503$
\end{tabular}

considerar apenas as que têm significado clínico, ou seja, a onda positiva mais evidente e as negativas anterior e posterior. As deflexões P55 e P63, respectivamente em cães e ovinos, registradas neste estudo possuem latência muito semelhante à P2, identificada por outros autores em cães (Strain et al. 1991b, Kimotsuki et al. 2005) e ovinos (Strain et al. 1991a). As latências médias da onda P2 registradas por Strain et al. (1991b) e por Kimotsuki et al. (2005) em cães foram de $54,5 \pm 7,4$ e $52,9 \pm 6,0 \mathrm{~ms}$, respectivamente. A latência de P2 observada em ovinos por Strain et al. (1991a) foi de $52,8 \pm 2,0 \mathrm{~ms}$, um pouco menor que o valor observado neste estudo.

A idade do animal é um fator não patológico a ser levado em consideração na interpretação dos resultados (Kimotsuki et al. 2006, Strain et al. 2006). Estudos mostraram diferenças na amplitude, morfologia e latência dos potenciais quando compararam resultados de animais recém-nascidos, jovens e adultos (Boyer \& Kirk 1973, Strain et al. 1991b, Takeuchi et al. 1993). Alterações no PEV também foram observadas em cães idosos (Kimotsuki et al. 2006). Por este motivo, apenas animais adultos foram incluídos neste estudo de normatização.

Outro fator que influencia diretamente o PEV, é o tamanho da cabeça (Strain et al. 2006). A variabilidade dos resultados desta padronização pode ser justificada pelo fato de os animais serem sem raça definida e, consequentemente, com diferentes tamanhos de cabeça. A variabilidade da amplitude das ondas também foi observada por outros autores, mas esta medida não tem significado clínico na interpretação do PEV (Sato et al. 1982, Strain et al. 1986b).

Neste estudo, os exames foram realizados apenas com contenção física dos animais. Alguns autores não conseguiram registrar o PEV de animais sem anestesia, devido à movimentação dos animais e presença de artefatos musculares (Bichsel et al. 1988). Diferentes anestésicos estão sendo utilizados para registro do PEV de animais, incluindo tiopental e halotano (Bichsel et al. 1988), metoxiflurano (Boyer \& Kirk 1973), xilazina (Kimotsuki et al. 2006) e isofluorano (Sims et al. 1989). No presente trabalho, não foi utilizada anestesia, porém, dos 20 cães e 20 ovinos tes- tados, apenas 17 de cada grupo foram utilizados no estudo normativo, pois o registro de três animais ficou com qualidade técnica ruim. Ao interpretar o resultado do PEV, é importante considerar se houve uso de anestésicos, pois a anestesia pode alterar os resultados de potenciais evocados (Chiappa 1990), principalmente causando prolongamento das latências registradas (Sims et al. 1989).

Muitos estudos de PEV em animais usam flashes como estímulo (Boyer \& Kirk 1973, Bichsel et al. 1988, Strain et al. 1991a, 2006, Kimotsuki et al. 2006). Este tipo de estímulo tem sido utilizado em medicina veterinária, pois requer menor cooperação do animal e não há necessidade de fixar a visão em um ponto específico, como é necessário com uso do estímulo com padrão reverso (Sims \& Laratta 1988).

Este estudo normativo é fundamental para comparação com PEV de animais com diferentes enfermidades que afetem a via visual central. O PEV já foi utilizado em medicina veterinária para avaliar a toxicidade de medicamentos (Sato 1985, Imai et al. 1990) e para diagnosticar alterações centrais em cães com distúrbios eletrolíticos (Lee et al. 1969). Alterações no PEV obtido de ovinos com scrapie já foram relatadas, incluindo diminuição significativa na amplitude das ondas (Strain et al. 1986a). Outro estudo também registrou alterações no PEV em ovinos com listeriose e em ruminantes com polioencefalomalácia (Strain et al. 1990).

\section{CONCLUSÕES}

Pode se concluir que é possível a obtenção de Potencial evocado visual (PEV) de cães e ovinos sem uso de sedação ou anestesia.

0 achado mais consistente em todos os exames foi uma deflexão eletropositiva, com latência média de 55,4ms em cães (P55) e 63,75ms em ovinos (P63).

Outras duas ondas eletronegativas também foram identificadas: N31 e N75 em cães; e N42 e N86 em ovinos.

Os valores de latência e amplitudes observados podem ser utilizados como referência para comparação com resultados obtidos de animais com diferentes doenças acometendo o sistema nervoso central, particularmente as vias visuais centrais.

Agradecimentos.- À Fundação de Amparo à Pesquisa do Estado de São Paulo (FAPESP) pelo suporte financeiro na forma de bolsa (Processo 2011/22769-9).

\section{REFERÊNCIAS}

Bichsel P., Oliver Jr J.E., Coulter D.B. \& Brown J. 1988. Recording of visual-evoked potentials in dogs with scalp electrodes. J. Vet. Intern. Med. 2:145-149.

Boyer S. \& Kirk G.R. 1973. Maturation of the visual evoked response in the dog. Exp. Neurol. 38:449-457.

Chiappa K.H. 1990. Evoked Potentials in Clinical Medicine. Raven Press, New York, p.37-171.

Creel D.J. 1973. Visually evoked responses in the rat, guinea pig, cat, monkey, and man. Exp. Neurol. 40:351-366.

Gregory N.G. \& Wotton S.B. 1983. Studies on the central nervous system: visually evoked cortical responses in sheep. Res. Vet. Sci. 34:315-319.

Imai R., Sugimoto S., Ando T. \& Sato S. 1990. A procedure for recording electroretinogram and visual evoked potential in freely moving cats. J. Toxicol. Sci. 15:263-274. 
Kimotsuki T., Yasuda M., Tamahara S., Matsuki N. \& Ono K. 2005. Topographic analysis of Flash visual evoked potentials in dogs. J. Vet. Med. Sci. 67:869-875.

Kimotsuki T., Yasuda M., Tamahara S., Tomihari M., Matsuki N. \& Ono K. 2006. Age-associated changes of flash visual evoked potentials in dogs. J. Vet. Med. Sci. 68:79-82.

Lee S.I., Klass D.W. \& Wakim K.G. 1969. Changes in visual evoked potentials induced by electrolyte alterations in dogs. Electroencephalogr. Clin. Neurophysiol. 27:677-678.

Loew E.R. 2005. Atividade elétrica do sistema visual: o ERG e a VER, p.1723. In: Riis R.C. (Ed.), Segredos em Oftalmologia de Pequenos Animais: respostas necessárias ao dia-a-dia. Artmed, Porto Alegre.

Michanek M. 2013. Visual Evoked Potentials (VEP): utveckling hos den växande hasten [In Swedish]. Institutionen för Kliniska Vetenskaper, Kurskod, Suécia. 31p.

Sato S. 1985. Toxic effects on the visual system of diaminodiphenoxybutane, quinine, and ethambutol in conscious dogs. Fundament. Appl. Toxicol. 5:777-784.

Sato S., Sugimoto S. \& Chiba S. 1982. A procedure for recording electroretinogram and visual evoked potential in conscious dogs. J. Pharmacol. Methods 8:173-181.

Sims M.H. \& Laratta L.J. 1988. Visual-evoked potentials in cats, using a light- emitting diode stimulator. Am. J. Vet. Res. 49:1876-1881.

Sims M.H., Laratta L.J., Bubb W.J. \& Morgan R.V. 1989. Waveform analysis and reproducibility of visual-evoked potentials in dogs. Am. J. Vet. Res. 50:1823-1828.
Strain G.M., Olcott B.M. \& Braun W.F. 1986a. Electroencephalogram and evoked potentials in naturally occurring scrapie in sheep. Am. J. Vet. Res. 47:828-836

Strain G.M., Olcott B.M. \& Hokett L.D. 1986b. Electroretinogram and visual-evoked potential measurements in Holstein cows. Am. J. Vet. Res. 47:1079-1081.

Strain G.M., Graham M.C., Claxton M.S. \& Olcott B.M. 1989. Postnatal development of brainstem auditory-evoked potentials, electroretinograms, and visual-evoked potentials in the calf. J. Vet. Intern. Med. 3:231-237.

Strain G.M., Claxton M.S., Olcott B.M. \& Turnquist S.E. 1990. Visual-evoked potentials and electroretinograms in ruminants with thiamine-responsive polioencephalomalacia or suspected listeriosis. Am. J. Vet. Res. 51:1513-1517.

Strain G.M., Claxton M.S., Prescott-Mathews J.S. \& LaPhand D.J. 1991a. Electroretinogram and visual-evoked potential measurements in sheep. Can. Vet. J. 55:1-4.

Strain G.M., Jackson R.M. \& Tedford B.L. 1991b. Postnatal development of the visual-evoked potential in dogs. Am. J. Vet. Res. 52:231-235.

Strain G.M., Tedford B.L. \& Gill M.S. 2006. Brainstem auditory evoked potentials and flash visual evoked potentials in Vietnamese miniature pot-bellied pigs. Res. Vet. Sci. 80:91-95.

Ström L. 2004. Visual Evoked Potentials in the Horse [In Swedish]. Department of Large Animal Medicine and Surgery, SLU, Uppsala. 44p.

Takeuchi T., Suzuki M., Sitizyo K. \& Saito T. 1993. Postnatal development of visual evoked potentials in Japanese Black Calves. Japan. J. Physiol. 43:809-815. 